\title{
Safety and efficacy of regional citrate anticoagulation in continuous blood purification treatment of patients with multiple organ dysfunction syndrome
}

\author{
B. Tuerdi ${ }^{1}$, L. Zuo ${ }^{1}$, H. Sun ${ }^{1}$, K. Wang ${ }^{1}$, Z. Wang ${ }^{2}$ and G. Li ${ }^{2}$ \\ ${ }^{1}$ Respiratory Intensive Care Units, the First Affiliated Hospital of Xinjiang Medical University, Urumqi, Xinjiang, China \\ ${ }^{2}$ Intensive Care Units, Branch of the First Affiliated Hospital of Xinjiang Medical University, Changji, Xinjiang, China
}

\begin{abstract}
The aim of this study was to discuss the safety and efficacy of regional citrate anticoagulation (RCA) on continuous blood purification (CBP) during the treatment of multiple organ dysfunction syndrome (MODS). Thirty-five patients with MODS were divided into two groups: the local citrate anticoagulation (RCA) group, and the heparin-free blood purification (hfBP) group. The MODS severity was assessed according to Marshall's MODS score criteria. Blood coagulation indicators, blood pressure, filter lifespan, filter replacement frequency, anticoagulation indicators, and main metabolic and electrolyte indicators were analyzed and compared between RCA and hfBP groups. RCA resulted in lower blood pressure than hfBP. The filter efficacy in RCA treatment was longer than in the hfBP group. The blood clearance of creatine, blood urea nitrogen and uric acid was better in the RCA group. RCA also led to higher $\mathrm{pH}$ than hfBP. Neither treatment resulted in severe bleeding events. In addition, MODS score was positively correlated with prothrombin time and activated partial thromboplastin time but negatively correlated with platelet concentration. RCA is a safer and more effective method in CBP treatment; however, it could also lead to low blood pressure and blood alkalosis.
\end{abstract}

Key words: Multiple organ dysfunction syndrome; Continuous blood purification; Blood coagulation function; Sodium citrate

\section{Introduction}

Multiple organ dysfunction syndrome (MODS) is a common critical illness, referring to the occurrence of simultaneous or sequential organ or system dysfunction $24 \mathrm{~h}$ after severe infection, wound shock, major surgery, severe pancreatitis, cardio-pulmonary resuscitation or other protopathies (1). It is generally believed that MODS is attributed to uncontrolled inflammatory mediators release, usually accompanied by inflammatory and metabolic disorders (2). Despite MODS pathogenesis being widely acknowledged and various therapies being introduced, the mortality of MODS is still much higher than $40 \%$, ranking first among all critical illness-caused deaths $(3,4)$.

Continuous blood purification (CBP), also named continuous renal replacement therapy, is a technology used to eliminate blood metabolites slowly and continually. As a new technology, CBP has already been applied in nonkidney disease treatments and has shown unique advantages when treating severe acute pancreatitis, systemic inflammatory response syndrome, sepsis, acute respiratory distress syndrome and MODS, becoming the main measure used to rescue critically ill patients (5). CBP is characterized by its hemodynamic stability, high metabolites clearance rate, efficiency of inflammatory mediator elimination and provision of nutritional support (6). In recent years, CBP has been widely applied to salvage and treat severely ill patients with remarkable therapeutic efficacy. CBP has been extensively applied in intensive care units in China, and its use on MODS treatment has been fully acknowledged (7). However, CBP needs continuous anticoagulation. Patients undergoing CBP are usually accompanied by active bleeding or a high risk of bleeding. The disseminated intravascular coagulation, triggered by severe wounds, infection, sepsis and shock, needs to be controlled (8). Insufficient anticoagulation (frequent coagulation) can suspend the treatment, and raise the clinical costs as well. On the other hand, over-anticoagulation may result in bleeding, or even life-threatening problems (9).

Therefore, the conduct of a safe and effective anticoagulation during CBP treatment is paramount. Although there are various anticoagulants available, none is omnipotent, given the variety of patients' physical conditions (10). For instance, the commonly used heparin may result

Correspondence: B. Tuerdi: <zhangjianxwhosp@126.com> 
in severe bleeding; low-molecular-weight heparin needs a high water-flow rate and the flow increases the volume load, which cannot be tolerated by hemodynamically instable patients; trisodium citrate is not consistent, and the technical aspect of its use is rather complicated; and regional citrate anticoagulation (RCA) could lead to low blood pressure of patients. The safety and efficacy of various anticoagulants need to be evaluated to provide useful information for clinicians when choosing the appropriate anticoagulants for their patients. On the other hand, heparin-free blood purification (hfBP) combined with molecular adsorbent recirculating system (MARS) showed a better non-bleeding outcome. In addition, heparin-freeMARS purification did not compromise the circuit function and longevity (11). A study on unfractionated heparin in 61 MARS treatment sessions in 33 patients showed that the platelet count was reduced but the platelet function remained unchanged (12). Heparin-free purification was also required for patients without disseminated intravascular coagulation, who demonstrated some coagulation abnormalities and thrombocytopenia during coupled plasma filtration adsorption (CFPA) purification therapy (13).

In this study, RCA and hfBP were compared in terms of the anticoagulation safety and efficacy during CBP treatment of MODS patients. This study may provide evidence that RCA could be a better choice for MODS CBP treatments.

\section{Material and Methods}

\section{Patients}

Thirty-five patients (25 males and 10 females) with MODS who came to the hospital for CBP during June 2009 and September 2010 were enrolled in this study. The MODS of the 35 patients was caused by trauma $(n=8)$, septic shock $(n=10)$, severe acute pancreatitis $(n=7)$, pregnancyinduced hypertension $(n=3)$, postpartum hemorrhage $(n=2)$, hepatic failure $(n=1)$, open-heart surgery $(n=3)$ and abdominal aortic aneurysm $(n=1)$. All the included participants who agreed with this study were randomly assigned to RCA blood purification (RCA group, $n=17)$ or the hfBP group $(n=18)$. Written informed consent and ethics approval were signed by the patients' legal representatives.

\section{Inclusion and exclusion criteria}

Patients with MODS who needed CBP and met the MODS diagnosis criteria (the Marshall 1995 MODS score criteria, shown in Supplementary Table S1) were included $(14,15)$. Those with MODS caused by hematologic system disorders or severe thrombocytopenia were excluded.

\section{Intervention}

Diapact polysulfone HI PS 18 filter (B. Braun, Germany) and circuit tube were used to establish the extracorporeal circuit system. The patients in both groups were treated with continuous veno-venous hemofiltration (CVVH). Pre-dilution mode was used and the replacement fluid speed was $3000-4000 \mathrm{~mL} / \mathrm{h}$. The blood flow was kept above $150 \mathrm{~mL} / \mathrm{min}$. Ionized calcium (iCa) levels were measured $1 \mathrm{~h}$ after the start of CVVH and every $6 \mathrm{~h}$ thereafter.

Anticoagulant citrate dextrose A (ACD-A) solution (600 $\mathrm{mL}$, purchased from Nigale Corporation, China) was used as the replacement fluid in the RCA group. The ACD-A infusion rate was $2-2.5 \%$ of blood flow rate, which was $>150 \mathrm{~mL} / \mathrm{min}$. During this procedure, the concentration of main electrolytes reached the final $140 \mathrm{mmol} / \mathrm{L}$ of $\mathrm{Na}^{+}, 105 \mathrm{mmol} / \mathrm{L}$ of $\mathrm{Cl}^{-}, 0 \mathrm{mmol}$ of $\mathrm{Ca}^{2+}, 0.94 \mathrm{of} \mathrm{Mg}^{2+}$, $11.1 \mathrm{mmol} / \mathrm{L}$ of glucose, $21 \mathrm{mmol} / \mathrm{L}$ of $\mathrm{HCO}_{3}{ }^{-}$and $4.6 \mathrm{mmol} / \mathrm{L}$ of citrate. Bicarbonate substitution fluid was used as the

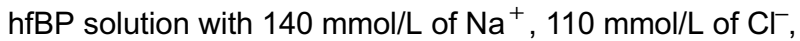

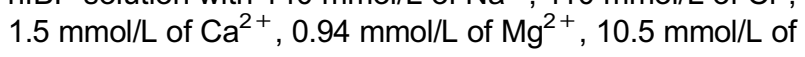
glucose and $35 \mathrm{mmol} / \mathrm{L}$ of $\mathrm{HCO}_{3}{ }^{-}$.

\section{Adverse reactions}

No reverse reactions were seen in either group. The evaluated reverse reactions include paresthesia, twitching movement, fever and bleeding complications.

\section{Study endpoints}

Our study endpoints were efficacy and safety. Safety was defined as the lack of adverse events such as hemorrhage complications including digestive tract, respiratory tract, incision, oral mucosa and derma hemorrhage before and after treatment. The duration of the first filter and the replacement frequency of filters were assessed. Major bleeding was defined as the presence of severe bleeding with spontaneous blood pressure drop. Metabolic alkalosis was defined as $\mathrm{pH}>7.5$. Hypocalcemia was defined as ionized calcium level $<1.1 \mathrm{mmol} / \mathrm{L}$.

\section{Data collection}

The severity of MODS was scored using MODS score system before and after the treatment in the two groups. The Glasgow Coma Scale was calculated by a nurse who did not participate the study. The hemorrhage complications were assessed by a physician who was not involved in this study.

Coagulation indicators including prothrombin time (PT), PT activity, activated partial thromboplastin time (APTT), international normalized ratio (INR), fibrinogen (FIB) and platelet concentrations were recorded before and $24 \mathrm{~h}$ after CBP. Besides, the pressure of artery (PA), the pressure before hemofilter (PBE), the pressure of vein $(\mathrm{PV})$, transmembrane pressure (TMP), the duration of filter, and the filter replacement frequency were also recorded by a physician. The $\mathrm{pH}$ and $\mathrm{iCa}$ levels of all participants were monitored four times every day. Electrolytes and platelet counts were measured daily. The level of sodium was also monitored to detect RCArelated electrolyte derangements. 


\section{Statistical analyses}

Data are reported as means \pm SD. Data were analyzed using the SPSS 20.0 package (IBM, USA). One-way ANOVA was used to compare differences of the indicators among different MODS groups before treatment. Parameters between the RCA and hfBP groups were compared using the $t$-test. Pre-treatment and post-treatment assessments were compared using the $t$-test. Bartlett sphericity test with or without Greenhouse-Geisser correction was performed to compare inter-group or intra-group data at different time points. Statistical significance was determined if $P$ values were less than 0.05 .

\section{Results}

Clinical characteristics of participants at the initiation of blood purification

Generally, there were no notable differences in the tested indicators between the RCA and hfBP groups (Table 1).

\section{Blood coagulation indicators}

In both groups, the coagulation indicators did not differ significantly before and after treatment. There was also no significant difference between the RCA post-treatment outcomes and the hfBP post-treatment outcomes (Table 2).

Hemorrhage events and blood pressure comparison

No patients in either group showed hemorrhage symptoms (Table 3). Within each group, PA did not differ significantly between different time points, whereas PBE, PV and TMP altered substantially as the anticoagulation treatment time advanced. Except for TMP, the remaining tested indicators were significantly higher in the hfBP group than in the RCA group. RCA resulted in lower blood pressure than hfBP treatment, indicating that RCA might be a risk factor for bleeding.

\section{Lifespan and replacement frequency of the filter}

The filter lifespan of the RCA group was approximately $29.8 \mathrm{~h}$, whereas that of the hfBP group was about $12.5 \mathrm{~h}$ and this difference was significant. The replacement frequency of the RCA group (5.90\%) was substantially smaller than that of the hfBP group (88.90\%). These results indicated that hfBP increased the risk of blood clotting, showing worse efficacy in anticoagulation (Table 4).

\section{Blood clearance}

The concentration of creatine $(\mathrm{Cr})$, blood urea nitrogen (BUN) and MODS score were significantly lower after both treatments compared with pre-treatment. $\mathrm{Cr}$ and uric acid concentrations in the hfBP group were both significantly higher after treatment than in the RCA group (Table 5). These results indicated that RCA had better clearance outcomes in terms of $\mathrm{Cr}$ and uric acid clearance than hfBP.

\section{Main metabolic and electrolyte indicators}

RCA significantly increased systemic $\mathrm{pH}$ after treatment, suggesting that RCA could result in metabolic alkalosis

Table 1. Baseline characteristics of patients with multiple organ dysfunction syndrome (MODS) at the initiation of blood purification.

\begin{tabular}{lcccc}
\hline Variable & RCA group & Heparin-free group & t/ $\chi 2$ & P value \\
\hline Age (years) & $52.75 \pm 21.44$ & $50.50 \pm 15.71$ & 0.29 & 0.77 \\
Gender (\%) & & & & \\
$\quad$ Male & 70.6 & 61.1 & 0.35 & 0.41 \\
$\quad$ Female & 29.4 & 38.9 & & \\
MODS score & $8.00 \pm 3.33$ & $9.39 \pm 2.25$ & -1.37 & 0.18 \\
PT (s) & $16.86 \pm 3.82$ & $17.79 \pm 2.44$ & -0.82 & 0.42 \\
PT activity $(\%)$ & $61.87 \pm 35.57$ & $49.18 \pm 11.53$ & 1.2 & 0.25 \\
APTT (s) & $54.40 \pm 53.76$ & $31.98 \pm 4.23$ & 1.44 & 0.18 \\
INR & $1.41 \pm 0.34$ & $1.54 \pm 0.22$ & -1.22 & 0.23 \\
FIB (g/L) & $5.29 \pm 1.65$ & $6.16 \pm 2.58$ & -1.03 & 0.31 \\
Platlet concentration (10 $/ \mathrm{L})$ & $116.17 \pm 74.25$ & $107.80 \pm 106.09$ & 0.24 & 0.82 \\
Sodium concentration $(\mathrm{mmol} / \mathrm{L})$ & $134.92 \pm 5.96$ & $137.67 \pm 6.52$ & -1.17 & 0.25 \\
Calcium concentration $(\mathrm{mmol} / \mathrm{L})$ & $1.98 \pm 1.10$ & $1.97 \pm 0.26$ & 0.58 & 0.97 \\
BUN concentration $(\mathrm{mmol} / \mathrm{L})$ & $25.69 \pm 22.08$ & $27.87 \pm 15.35$ & -0.32 & 0.75 \\
Creatine concentration $(\mathrm{mmol} / \mathrm{L})$ & $423.00 \pm 472.64$ & $439.67 \pm 219.31$ & -0.11 & 0.91 \\
UAC Concentration $(\mathrm{mmol} / \mathrm{L})$ & $338.67 \pm 204.91$ & $365.94 \pm 189.66$ & -0.37 & 0.71 \\
\hline
\end{tabular}

Data are reported as means \pm SD or number and percentage. RCA: regional citrate anticoagulation; PT: prothrombin time; APTT: activated partial thromboplastin time; INR: international normalized ratio; FIB: fibrinogen; BUN: blood urea nitrogen; UAC: uric acid. Statistical analysis was performed with the $t$-test or chi-square test. 
Table 2. Coagulation indicators at baseline and after continuous blood purification within regional citrate anticoagulation (RCA) or heparin-free blood purification (hfBP) groups.

\begin{tabular}{lcccccc}
\hline & PT $(\mathrm{s})$ & PT activity $(\%)$ & APTT $(\mathrm{s})$ & INR & FIB $(\mathrm{g} / \mathrm{L})$ & Platelet concentration $\left(10^{9} / \mathrm{L}\right)$ \\
\hline RCA group & & & & & & \\
$\quad$ Before treatment & $16.86 \pm 3.82$ & $61.87 \pm 35.57$ & $54.40 \pm 53.76$ & $1.14 \pm 0.34$ & $5.29 \pm 1.65$ & $116.17 \pm 74.25$ \\
After treatment & $17.43 \pm 2.65$ & $48.58 \pm 11.88$ & $33.07 \pm 5.37$ & $1.46 \pm 0.23$ & $4.41 \pm 1.80$ & $119.83 \pm 62.72$ \\
$t$ & -0.49 & 1.43 & 1.41 & -0.45 & 1.34 & -0.19 \\
P value & 0.66 & 0.18 & 0.19 & 0.66 & 0.21 & 0.85 \\
hfBP group & & & & & & $107.80 \pm 106.09$ \\
$\quad$ Before treatment & $17.79 \pm 2.44$ & $49.18 \pm 11.53$ & $31.98 \pm 4.23$ & $1.54 \pm 0.22$ & $6.16 \pm 2.58$ & $102.64 \pm 112.50$ \\
After treatment & $17.71 \pm 3.73$ & $50.32 \pm 9.95$ & $34.56 \pm 8.15$ & $1.55 \pm 0.48$ & $5.65 \pm 2.24$ & 0.78 \\
$t$ & 0.78 & -0.38 & -1.8 & -0.14 & 1.1 & 0.45 \\
P value & 0.94 & 0.71 & 0.09 & 0.89 & 0.29 & \\
\hline
\end{tabular}

Data are reported as means \pm SD. PT: prothrombin time; APTT: activated partial thromboplastin time; INR: international normalized ratio; FIB: fibrinogen. Statistical analysis was performed with the $t$-test.

Table 3. Anticoagulation efficacy of regional citrate anticoagulation (RCA) and heparin-free blood purification (hfBP) treatments.

\begin{tabular}{|c|c|c|c|c|c|c|c|c|c|c|c|}
\hline \multirow[t]{2}{*}{ Group } & \multirow[t]{2}{*}{$0 \mathrm{~h}$} & \multirow[t]{2}{*}{$2 \mathrm{~h}$} & \multirow[t]{2}{*}{$4 \mathrm{~h}$} & \multirow[t]{2}{*}{$6 \mathrm{~h}$} & \multirow[t]{2}{*}{$8 \mathrm{~h}$} & \multirow[t]{2}{*}{$10 \mathrm{~h}$} & \multirow[t]{2}{*}{$12 \mathrm{~h}$} & \multicolumn{2}{|c|}{ F value } & \multicolumn{2}{|c|}{$P$ value } \\
\hline & & & & & & & & Inter-class & Intra-class & Inter-class & Intra-class \\
\hline \multicolumn{12}{|l|}{ PA } \\
\hline $\mathrm{RCA}$ & $53.42 \pm 17.63$ & $49 \pm 18.21$ & $47.5 \pm 16.34$ & $47.08 \pm 17.29$ & $45.25 \pm 11.59$ & $48.33 \pm 13.51$ & $50.25 \pm 20.56$ & 21.04 & 0.69 & 0.00 & 0.59 \\
\hline hfBP & $83.50 \pm 40.31$ & $74.11 \pm 29.81$ & $86.56 \pm 37.91$ & $83.06 \pm 29.32$ & $80.83 \pm 26$ & $75.61 \pm 21$ & $88.89 \pm 37.86$ & & & & \\
\hline \multicolumn{12}{|l|}{ PBE } \\
\hline $\mathrm{RCA}$ & $99.08 \pm 35.08$ & $106.08 \pm 27.17$ & $116.58 \pm 23.71$ & $112.25 \pm 23.25$ & $112.92 \pm 23.99$ & $121.92 \pm 47.97$ & $141.83 \pm 83.95$ & 16.09 & 6.46 & 0.00 & 0.00 \\
\hline hfBP & $147.39 \pm 46.31$ & $146.78 \pm 36.07$ & $163.33 \pm 71.02$ & $173.33 \pm 58.57$ & $199.11 \pm 74.33$ & $228.11 \pm 106.7$ & $239.5 \pm 117.96$ & & & & \\
\hline \multicolumn{12}{|c|}{ PV } \\
\hline RCA & $43.17 \pm 19.31$ & $43 \pm 14.69$ & $49.58 \pm 16.21$ & $47.42 \pm 15.58$ & $47.5 \pm 15.83$ & $90.67 \pm 173.77$ & 7 $59.33 \pm 37$ & 8.00 & 3.73 & 0.00 & 0.04 \\
\hline hfBP & $71.17 \pm 29.82$ & $73 \pm 25.56$ & $76.67 \pm 28.17$ & $79.72 \pm 35.66$ & $103.72 \pm 63.42$ & $121.33 \pm 80.81$ & $128.89 \pm 91.77$ & & & & \\
\hline \multicolumn{12}{|l|}{ TMP } \\
\hline RCA & $40.67 \pm 14.44$ & $69.17 \pm 26.61$ & $75.25 \pm 14.12$ & $80.92 \pm 17$ & $84.08 \pm 15.55$ & $92.42 \pm 43.72$ & $117.08 \pm 89.33$ & 0.78 & 15.86 & 0.39 & 0.00 \\
\hline hfBP & $46.39 \pm 32.89$ & $60.22 \pm 20.6$ & $69.5 \pm 26.01$ & $97.89 \pm 56.44$ & $105.17 \pm 66.77$ & $122.78 \pm 69.65$ & $135.89 \pm 72.99$ & & & & \\
\hline
\end{tabular}

Data are reported as means \pm SD. PA: pressure of artery; PBE: pressure before hemofilter; PV: pressure of vein; TMP: transmembrane pressure. Statistical analysis was performed with one-way ANOVA.

Table 4. Filter lifespan and the number of patients with multiple organ dysfunction syndrome treated with regional citrate anticoagulation (RCA) or heparin-free blood purification (hfBP) undergoing filter replacement.

\begin{tabular}{lcccccc}
\hline Group & $\begin{array}{c}\text { Filter } \\
\text { lifespan }(\mathrm{h})\end{array}$ & $\begin{array}{c}\text { Complete } \\
\text { replacement }(\mathrm{n})\end{array}$ & $\begin{array}{c}\text { Filter } \\
\text { replacement }(\mathrm{n})\end{array}$ & $\begin{array}{c}\text { No } \\
\text { replacement }(\mathrm{n})\end{array}$ & $\begin{array}{c}\text { Total } \\
(\mathrm{n})\end{array}$ & $\begin{array}{c}\text { Replacement } \\
\text { rate }(\%)\end{array}$ \\
\hline RCA & $29.84 \pm 21.34$ & 0 & 2 & 15 & 17 & 5.90 \\
HfBP & $12.46 \pm 17.13$ & 15 & 1 & 2 & 18 & $88.9^{*}$ \\
& & $\chi 2=20.91$ & $\mathrm{P}=0.001$ & & \\
\hline
\end{tabular}

Data are reported as means $\pm S D$. * $P<0.05$, hfBP vs RCA group (chi-square test).

during treatment. RCA significantly decreased iCa level after treatment, suggesting that RCA could result in hypocalcemia. $\mathrm{Na}^{+}$and $\mathrm{HCO}_{3}{ }^{-}$concentrations had no significant difference before treatment and after treatment in both groups. Similarly, RCA also showed significantly higher $\mathrm{pH}$ and lower iCa level than hfBP treatment. $\mathrm{Na}^{+}$and $\mathrm{HCO}_{3}^{-}$concentrations had no significant difference between groups (Table 6).

\section{Correlation between coagulation indicators and MODS scores}

PT and INR showed no difference among the three MODS score groups. PT activity in the $>10-15$ group was more intense than the $0-\leqslant 5$ and $>5-\leqslant 10$ groups. APTT of the $>5-\leqslant 10$ group was significantly lower than in the $0-\leqslant 5$ and $>10-15$ groups, and platelet concentration of the $>10-15$ group was significantly lower than the $0-\leqslant 5$ 
Table 5. Blood clearance parameters and multiple organ dysfunction syndrome (MODS) scores of regional citrate anticoagulation (RCA) and heparin-free blood purification (hfBP) treatment groups.

\begin{tabular}{lccccc}
\hline Variable & \multicolumn{2}{c}{ RCA group } & & \multicolumn{2}{c}{ hfBP group } \\
\cline { 2 - 3 } \cline { 5 - 6 } \cline { 5 - 6 } & Pre-treatment & Post-treatment & & Pre-treatment & Post-treatment \\
\hline Creatine $(\mu \mathrm{mol} / \mathrm{L})$ & $423 \pm 462.25$ & $136 \pm 104.01^{*}$ & & $439.67 \pm 216.15$ & $292.72 \pm 157.42^{* \Delta}$ \\
BUN $(\mathrm{mmol} / \mathrm{L})$ & $25.69 \pm 22.08$ & $11.38 \pm 10.26^{*}$ & & $27.87 \pm 15.35$ & $15.91 \pm 9.14^{*}$ \\
UAC $(\mu \mathrm{mol} / \mathrm{L})$ & $338.67 \pm 204.91$ & $127.50 \pm 75.01$ & & $365.94 \pm 189.66$ & $214.83 \pm 123.58^{* \Delta}$ \\
MODS score & $8.00 \pm 3.33$ & $6.33 \pm 2.61^{*}$ & & $9.39 \pm 2.25$ & $8.11 \pm 2.89^{*}$ \\
\hline
\end{tabular}

Data are reported as means $\pm S D$. BUN: blood urea nitrogen; UAC: uric acid. ${ }^{*} P<0.05$, post-treatment $v s$ pre-treatment; ${ }^{\Delta} \mathrm{P}<0.05$, heparin-free $v s \mathrm{RCA}$ after treatment. Statistical analysis was performed with the $t$-test.

Table 6. Main metabolic and electrolyte parameters before and after the treatment of patients with multiple organ dysfunction syndrome treated with regional citrate anticoagulation (RCA) or heparin-free blood purification (hfBP).

\begin{tabular}{lcccc}
\hline Group & $\mathrm{pH}$ & $\mathrm{iCa}(\mathrm{mmol} / \mathrm{L})$ & $\mathrm{Na}^{+}(\mathrm{mmol} / \mathrm{L})$ & $\mathrm{HCO}_{3}^{-}(\mathrm{mmol} / \mathrm{L})$ \\
\hline RCA & & & & \\
Before treatment & $7.35 \pm 0.14$ & $1.68 \pm 2.26$ & $134.92 \pm 5.96$ & $21.32 \pm 7.03$ \\
After treatment & $7.43 \pm 0.05^{\star+}$ & $1.08 \pm 0.10$ & $137.75 \pm 3.31$ & $24.16 \pm 1.74$ \\
hfBP & & & & \\
Before treatment & $7.42 \pm 0.09$ & $1.07 \pm 0.15$ & $137.67 \pm 6.52$ & $21.73 \pm 4.38$ \\
After treatment & $7.44 \pm 0.06$ & $1.06 \pm 0.14$ & $138.44 \pm 4.64$ & $22.69 \pm 2.70$ \\
\hline
\end{tabular}

Data are reported as means $\pm S D$. iCa: ionized Ca. ${ }^{*} P<0.05$, before-treatment $v s$ after-treatment group; ${ }^{+} \mathrm{P}<0.05$, RCA vs hfBP group. Statistical analysis was performed with the $t$-test.

Table 7. Correlation between multiple organ dysfunction syndrome (MODS) score and coagulation indicators of patients with multiple organ dysfunction syndrome treated with regional citrate anticoagulation or heparin-free blood purification.

\begin{tabular}{lclllll}
\hline MODS score & PT $(\mathrm{s})$ & PT activity $(\%)$ & APTT $(\mathrm{s})$ & INR & FIB $(\mathrm{g} / \mathrm{L})$ & Platelet $\left(10^{9} / \mathrm{L}\right)$ \\
\hline $0-\leqslant 5$ & $19.37 \pm 2.22$ & $41.70 \pm 8.41$ & $41.77 \pm 4.54$ & $1.62 \pm 0.21$ & $3.59 \pm 1.62$ & $130.00 \pm 79.89$ \\
$>5-\leqslant 10$ & $17.62 \pm 2.96$ & $50.45 \pm 14.11$ & $33.78 \pm 8.77$ & $1.51 \pm 0.25$ & $6.23 \pm 2.31^{*}$ & $129.19 \pm 99.81$ \\
$>10-\leqslant 15$ & $16.00 \pm 3.02$ & $70.52 \pm 41.50^{*+}$ & $61.09 \pm 68.26^{+}$ & $1.37 \pm 0.32$ & $5.58 \pm 1.75$ & $51.51 \pm 38.86^{+}$ \\
\hline
\end{tabular}

Data are reported as means \pm SD. PT: prothrombin time; APTT: activated partial thromboplastin time; INR: international normalized ratio; FIB: fibrinogen. ${ }^{*} \mathrm{P}<0.05$, before-treatment $v s$ after-treatment group, ${ }^{+} \mathrm{P}<0.05, \mathrm{RCA}$ vs hfBP group. Statistical analysis was performed with one-way ANOVA.

and $>5-\leqslant 10$ groups. FIB concentration in the $>5-\leqslant 10$ group was significantly more intense than in the $0-\leqslant 5$ group. These results indicated a positive correlation between PT activity and MODS score but a negative correlation between platelet concentration and MODS score (Table 7).

\section{Discussion}

CBP appears to be the last resort to rescue MODS patients due to its advantage of continuity during the treatment. In this process, protecting patients from bleeding is important. However, how to conduct anticoagulation during CBP therapy for MODS patients with a high risk for bleeding still remains problematic (9). We assessed the efficacy and safety of RCA in the CBP treatment in this study. We compared the anticoagulation effects of MODS patients going through RCA or hfBP. By analyzing blood coagulation indicators, blood pressure, blood clearance and the occurrence of complications, we arrived at the conclusion that RCA is a comparatively safe and effective method in CBP treatment of MODS patients. 
In our research, we selected ACD-A, a commercially available blood preservation solution, to act as the anticoagulant. There are many indicators to assess anticoagulation efficacy including whole blood activated clotting time, calcium level at the vein side of extracorporeal circulation and the lifespan of filters (16). We chose the lifespan of filters as our primary outcome of efficacy. A longer filter lifespan means a more effective treatment time and less treatment interruption. In our research, a better efficacy of RCA anticoagulation was found. This result was consistent with a published study, in which RCA treatment was related to a longer lifespan of filters compared with the control group (17). Morabito et al. (18) also suggested that RCA could ensure enough filter life and decrease treatment cost.

RCA technology inputs sodium citrate through arterial blood. Citrate and calcium react in the blood and form calcium citrate, which is hard to dissolve, so the activated calcium ions in the blood significantly decrease, thrombin and other blood coagulation processes occur leading to full anticoagulation. The most important aim of hemodialysis is to remove the poisonous solute and metabolites in the patients' bodies, and it has to be achieved only under the condition of efficient extra-corporeal circuit anticoagulation. Despite the reported efficacy of RCA, several safety endpoints should also be considered. The blood concentration of $\mathrm{Cr}, \mathrm{BUN}$, and UAC also significantly decreased after RCA treatment. Compared with the control group, RCA revealed better ability in $\mathrm{Cr}$, BUN and UAC clearance, indicating its higher safety. Citrate cannot be used with hemofiltration alone because of the insufficient clearance of sodium citrate in blood, which could cause the imbalance of serum sodium and bicarbonate concentrations (19). Moreover, high ultrafiltration rate was reported to reach high clearances (19). No report has carefully elaborated the mechanism by which RCA eliminates $\mathrm{Cr}$, BUN and UAC in blood after the blood purification process. However, studies found that citrate anti-coagulation indeed demonstrated better blood solute clearance than other purification methods (20-22). It has been agreed that the excess citrate used in RCA purification can be metabolized via the kidney and liver. We believe that this is related to the good $\mathrm{Cr}$, BUN and UAC clearance results of RCA purification method.

In the present study, we also assessed the blood pressure-related indicators, such as PA, PBE, PV and TMP, to evaluate the safety of different anticoagulants. RCA resulted in lower blood pressure than hfBP, suggesting a lower risk of bleeding. (23). The most common complication of RCA treatment is hypocalcemia, which together with metabolic alkalosis are closely correlated with the use of sodium citrate. In our study, hemorrhage complications were not observed in RCA and hfBP groups; however, it was possible that other complications such as citrate acidosis, hypernatremia and metabolic acid-basic disorder may occur (24). For example, the electrolyte indicator " $\mathrm{pH}$ " was higher in patients treated with RCA, suggesting that RCA may result in metabolic alkalosis. However, the risk of metabolic alkalosis when using citrate and bicarbonate is rarely high and could be lower when using a carefully calculated bicarbonate continuous veno-venous hemofiltration algorithm $(18,25)$. Another study pointed out that a lower dialysate bicarbonate concentration $(<25 \mathrm{mmol} / \mathrm{L})$ may lower the occurrence of alkalosis (26). As mentioned before, the severest complication caused by citrate infusion is hypocalcemia, which could result in myocardial dysfunction or even death $(27,28)$. The metabolic process of citrate in the body is fast. If we stop the input of sodium citrate, within 10 to 30 minutes the level of citrate and free calcium in the body can be restored to the normal level. Therefore, the proper operation may ensure safety in therapy. For example, metabolic complications could be reduced if a standardized protocol is applied to adjust dialysate flow and calcium substitution so that the blood $\mathrm{pH}$ and ionized calcium levels would be maintained within a normal range (29). In the present study, imbalances of iCa were not significantly different between the RCA and hfBP groups after treatment, which is probably because of the small study population. This contradiction requires a further study involving a larger patient cohort to study the ionized calcium imbalance after the treatment of RCA and other anticoagulants.

There are some limitations to this study. First, the sample consisted of only 35 patients, which was limited compared with other studies probing into RCA (30). Second, although this study included a variety of outcomes, it still lacked some important indicators, such as other potential complications like 30-day survival rate (31). Hence, we failed to present an impressive reduction in mortality after the use of RCA. The efficacy and safety of other anticoagulants could also be considered in further studies.

In conclusion, RCA treatment demonstrated safer and more effective outcomes than the hfBP treatment. However, RCA could also lead to several adverse effects such as blood alkalosis and low blood pressure.

\section{Supplementary material}

Click here to view [pdf].

\section{Acknowledgments}

This research was supported by the Natural Science Foundation of Xinjiang (\#2016D01C255). 


\section{References}

1. Walsh CR. Multiple organ dysfunction syndrome after multiple trauma. Orthop Nurs 2005; 24: 324-333; quiz 334-335.

2. Schmidt H, Lotze U, Ghanem A, Anker SD, Said SM, BraunDullaeus $\mathrm{R}$, et al. Relation of impaired interorgan communication and parasympathetic activity in chronic heart failure and multiple-organ dysfunction syndrome. J Crit Care 2014; 29: 367-373, doi: 10.1016/j.jcrc.2013.12.015.

3. Kochanek KD, Murphy SL, Anderson RN, Scott C. Deaths: final data for 2002. Natl Vital Stat Rep 2004; 53: 1-115.

4. Kung HC, Hoyert DL, Xu J, Murphy SL. Deaths: final data for 2005. Natl Vital Stat Rep 2008; 56: 1-120.

5. Rico MP, Fernandez Sarmiento J, Rojas Velasquez AM, Gonzalez Chaparro LS, Gastelbondo Amaya R, Mulett Hoyos $\mathrm{H}$, et al. Regional citrate anticoagulation for continuous renal replacement therapy in children. Pediatr Nephrol 2017; 32: 719, doi: 10.1007/s00467016-3568-1.

6. Kielstein JT, Kretschmer U, Ernst T, Hafer C, Bahr MJ, Haller $\mathrm{H}$, et al. Efficacy and cardiovascular tolerability of extended dialysis in critically ill patients: a randomized controlled study. Am J Kidney Dis 2004; 43: 342-349, doi: 10.1053/ j.ajkd.2003.10.021.

7. Kumar VA, Craig M, Depner TA, Yeun JY. Extended daily dialysis: A new approach to renal replacement for acute renal failure in the intensive care unit. Am J Kidney Dis 2000; 36: 294-300, doi: 10.1053/ajkd.2000.8973.

8. Baglin T. Disseminated intravascular coagulation: diagnosis and treatment. BMJ 1996; 312: 683-687, doi: 10.1136/ bmj.312.7032.683.

9. Berbece AN, Richardson RM. Sustained low-efficiency dialysis in the ICU: cost, anticoagulation, and solute removal. Kidney Int 2006; 70: 963-968, doi: 10.1038/sj.ki.5001700.

10. Pannu N, Gibney RN. Renal replacement therapy in the intensive care unit. Ther Clin Risk Manag 2005; 1: 141-150, doi: $10.2147 / \mathrm{tcrm} .1 .2 .141 .62908$.

11. Tan HK, Yang WS, Chow P, Lui HF, Choong HL, Wong KS. Anticoagulation minimization is safe and effective in albumin liver dialysis using the molecular adsorbent recirculating system. Artif Organs 2007; 31: 193-199, doi: 10.1111/j.15251594.2007.00364.x.

12. Faybik P, Bacher A, Kozek-Langenecker SA, Steltzer $H$, Krenn CG, Unger $S$, et al. Molecular adsorbent recirculating system and hemostasis in patients at high risk of bleeding: an observational study. Crit Care 2006; 10: R24, doi: 10.1186/ cc3985.

13. Abdul Cader R, Abdul Gafor H, Mohd R, Yen Kong W, Arshad N, Kong N. Coupled Plasma Filtration and Adsorption (CPFA): A Single Center Experience. Nephrourol Mon 2013; 5: 891-896, doi: 10.5812/numonthly.11904.

14. Gasparovic V, Filipovic-Grcic I, Merkler M, Pisl Z. Continuous renal replacement therapy (CRRT) or intermittent hemodialysis (IHD) - what is the procedure of choice in critically ill patients? Ren Fail 2003; 25: 855-862, doi: 10.1081/JDI-120024300.

15. Xing R, Jin Y, Sun L, Yang L, Li C, Li Z, et al. Interleukin-21 induces migration and invasion of fibroblast-like synoviocytes from patients with rheumatoid arthritis. Clin Exp Immunol 2016; 184: 147-158, doi: 10.1111/cei.12751.
16. Vargas Hein O, Kox WJ, Spies C. Anticoagulation in continuous renal replacement therapy. Contrib Nephrol 2004; 144: 308-316, doi: 10.1159/000078898.

17. Oudemans-van Straaten HM. Citrate anticoagulation for continuous renal replacement therapy in the critically ill. Blood Purif 2010; 29: 191-196, doi: 10.1159/000245646.

18. Morabito S, Pistolesi V, Tritapepe L, Zeppilli L, Polistena F, Strampelli E, et al. Regional citrate anticoagulation in cardiac surgery patients at high risk of bleeding: a continuous veno-venous hemofiltration protocol with a low concentration citrate solution. Crit Care 2012; 16: R111, doi: $10.1186 /$ cc11403.

19. Palsson R, Niles JL. Regional citrate anticoagulation in continuous venovenous hemofiltration in critically ill patients with a high risk of bleeding. Kidney Int 1999; 55: 1991-1997, doi: 10.1046/j.1523-1755.1999.00444.x.

20. Schmitz M, Taskaya G, Plum J, Hennersdorf M, Sucker C, Grabensee B, et al. Continuous venovenous haemofiltration using a citrate buffered substitution fluid. Anaesth Intensive Care 2007; 35: 529-535.

21. Onishi S, Yoshino S. Cathartic-induced fatal hypermagnesemia in the elderly. Intern Med 2006; 45: 207-210, doi: 10.2169/internalmedicine.45.1482.

22. Monchi M, Berghmans D, Ledoux D, Canivet JL, Dubois B, Damas P. Citrate vs. heparin for anticoagulation in continuous venovenous hemofiltration: a prospective randomized study. Intensive Care Med 2004; 30: 260-265, doi: 10.1007/ s00134-003-2047-x

23. Amanzadeh J, Reilly RF, Jr. Anticoagulation and continuous renal replacement therapy. Semin Dial 2006; 19: 311-316, doi: 10.1111/j.1525-139X.2006.00178.x.

24. Kramer L, Bauer E, Joukhadar C, StrobI W, Gendo A, Madl C, et al. Citrate pharmacokinetics and metabolism in cirrhotic and noncirrhotic critically ill patients. Crit Care Med 2003; 31: 2450-2455, doi: 10.1097/01.CCM.0000084871.76568.E6.

25. Hetzel GR, Schmitz M, Wissing H, Ries W, Schott G, Heering PJ. Regional citrate versus systemic heparin for anticoagulation in critically ill patients on continuous venovenous haemofiltration: a prospective randomized multicentre trial. Nephrol Dial Transplant 2011; 26, doi: 10.1093/ndt/gfq575.

26. Tolwani AJ, Prendergast MB, Speer RR, Stofan BS, Wille $\mathrm{KM}$. A practical citrate anticoagulation continuous venovenous hemodiafiltration protocol for metabolic control and high solute clearance. Clin J Am Soc Nephrol 2006; 1: 79-87, doi: 10.2215/CJN.00040505.

27. Zaloga GP. Hypocalcemia in critically ill patients. Crit Care Med 1992; 20: 251-262, doi: 10.1097/00003246-19920200000014.

28. Saner FH, Treckmann JW, Geis A, Losch C, Witzke O, Canbay A, et al. Efficacy and safety of regional citrate anticoagulation in liver transplant patients requiring postoperative renal replacement therapy. Nephrol Dial Transplant 2012; 27: 1651-1657, doi: 10.1093/ndt/gfr510.

29. Stucker F, Ponte B, Tataw J, Martin PY, Wozniak H, Pugin J, et al. Efficacy and safety of citrate-based anticoagulation compared to heparin in patients with acute kidney injury requiring continuous renal replacement therapy: a randomized controlled trial. Crit Care 2015; 19: 91, doi: 10.1186/ s13054-015-0822-z. 
30. Slowinski $T$, Morgera $S$, Joannidis $M$, Henneberg $T$, Stocker R, Helset E, et al. Safety and efficacy of regional citrate anticoagulation in continuous venovenous hemodialysis in the presence of liver failure: the Liver Citrate Anticoagulation Threshold (L-CAT) observational study. Crit Care 2015; 19: 349, doi: 10.1186/s13054015-1066-7.

31. Kawarazaki H, Uchino S. Validity of low-efficacy continuous renal replacement therapy in critically ill patients. Anaesthesiol Intensive Ther 2016; 48: 191-196, doi: 10.5603/AIT.a2016.0029. 